\title{
EFFECT OF INTERFACE ON THE SPINODAL DECOMPOSITION OF GaInPAs SOLID SOLUTIONS DURING LIQUID PHASE HETEROEPITAXY
}

\author{
J.M. OLCHOWIK \\ Technical University of Lublin, Department of Physics \\ Nadbystrzycka 38, 20-618 Lublin, Poland
}

\begin{abstract}
The area of thermodynamical instability in non-ideal solutions appears below a critical temperature. On exceeding this temperature, the excessive energy of the solution takes positive values. It means that the repulsive forces acting between solution elements begin to dominate which leads to the spinodal decomposition. In the present paper, the effect of interatomic interactions on the limits of spinodal decomposition of substitutional solutions of $\mathrm{Ga}-\mathrm{In}-\mathrm{P}-\mathrm{As}$ compounds was examined. The influence of the interface energy on the nature of spinodal decomposition was also analysed within the approximation of the coherent coupling of phases during heteroepitaxy from the liquid phase. It was demonstrated that the energy of elastic deformation of the interface lattice markedly changed conditions of the thermodynamical equilibrium.
\end{abstract}

PACS numbers: $81.10 . \mathrm{Dn}$

\section{Introduction}

In non-ideal solutions, during temperature reduction, the thermodynamical instability can be expected for a range of chemical compositions. It means, in accordance with the quasi-chemical approximation of solutions [1] that the repulsive forces act between components of solid solution which leads to the so-called spinodal decomposition.

The general theory has not been developed yet, therefore the published qualitative data on the range of instability show significant discrepancy depending on the assumed approximation [2, 3]. In general, the Gibbs' energy $\left(G^{m}\right)$ of the solution can be described using its configuration enthalpy $\left(H^{m}\right)$ and entropy $\left(S^{m}\right)$, thus

$$
G^{m}=H^{m}-T S^{m} .
$$

The configuration enthalpy of melting has always positive values and as a consequence of this, the value of Gibbs' energy is lower than the energy of the enthalpy term $H^{m}$. The incompatibility problem of the solution compounds is therefore 
reduced to the question about the value of Gibbs' energy in the entire range of solid solution compositions. The thermodynamical instability is expected in some range of compositions if this energy has the negative curvature.

\section{Derivation of instability limits for $A_{x} B_{1-x} C_{y} D_{1-y}$ type compounds}

In general the stability criterion states that the surface of Gibbs' thermodynamical potential should be convex in the direction of the concentration axes of constituents. Any fluctuaction in chemical compositions requires an increase in the free energy of the system. For the binary solutions the sufficient condition is given by the expression

$$
\partial^{2} G / \partial x^{2} \geq 0 \text {. }
$$

The ternary solutions of the $\mathrm{A}_{x} \mathrm{~B}_{1-x} \mathrm{C}$ and $\mathrm{AC}_{y} \mathrm{D}_{1-y}$ types can be described in a similar way considering them as pseudo-binary systems. The description of the second type quaternary solutions is much more difficult. The simultaneous substitution of atoms in both sublattices greatly complicates the nature of their interactions. The essence of the problem in determing the instability boundaries of the solution is the accepted way of representing Gibbs' potential as a function of molar composition.

According to the one of these approximations, the so-called regular approximation, the energy $G^{m}$ can be expressed as [4]

$$
\begin{aligned}
G^{m} & =R T \ln [x \ln x+y \ln y+(1-x) \ln (1-x)+(1-y) \ln (1-y)] \\
& +\alpha_{12}^{\mathbf{s}} x(1-x)+\alpha_{34}^{\mathbf{s}} y(1-y)+\alpha_{c}\left(x_{14}^{\mathbf{s}} x_{23}^{\mathbf{s}}-x_{13}^{\mathbf{s}} x_{24}^{\mathbf{s}}\right) ;
\end{aligned}
$$

where: $x_{i j}$ - component concentrations in a III-V solution, $\alpha_{i j}^{s}$ - interaction parameters in anion and cation sublattices, $\alpha_{\mathrm{c}}^{\mathrm{s}}=\alpha_{13}^{\mathrm{s}}+\alpha_{24}^{\mathrm{s}}-\alpha_{23}^{\mathrm{s}}-\alpha_{14}^{\mathrm{s}}$. It follows from the above that the area of thermodynamical instability depends on the value of the interaction parameter.

The spinodal decomposition should be expected for atomical configurations with dominating mutual repulsive forces. In order to express precisely Gibbs' energy in terms of molar compositions, the anion-cation pair concentration should be related with the $x$ and $y$ values. Knowing the number of moles $n=n_{1}+n_{2}=$ $n_{3}+n_{4}=n_{13}+n_{14}+n_{23}+n_{24}$, the concentrations of binary and ternary compounds can be described. Assuming a statistical distribution of anion-cation pairs in ternary solutions of $x, y$ molar composition, it can be proved that [5]

$$
\begin{aligned}
& G^{m}(x, y)=R T \ln [x \ln x+y \ln y+(1-x) \ln (1-x)+(1-y) \ln (1-y)] \\
& \quad+\alpha_{12}^{\mathrm{s}} x(1-x)+\alpha_{34}^{\mathrm{s}} y(1-y)+\alpha_{\mathrm{c}}^{\mathrm{s}}[x y-0.5(x+y)] .
\end{aligned}
$$

Taking these assumptions into account, the spinodal equation takes form [6]

$$
\left(\partial^{2} G^{m} / \partial x^{2}\right)\left(\partial^{2} G^{m} / \partial y^{2}\right)-\left(\partial^{2} G^{m} / \partial x \partial y\right)^{2}=0
$$

where

$$
\left(\partial^{2} G^{m} / \partial x^{2}\right)=R T /[x(1-x)]-2\left[y \alpha_{1}^{\mathrm{s}}+(1-y) \alpha_{2}^{\mathrm{s}}\right],
$$




$$
\begin{aligned}
& \left(\partial^{2} G^{m} / \partial y^{2}\right)=R T /[y(1-y)]-2\left[x \alpha_{3}^{\mathrm{s}}+(1-x) \alpha_{4}^{\mathrm{s}}\right] \\
& \left(\partial^{2} G^{m} / \partial x \partial y\right)=(1-2 x)\left(\alpha_{1}^{s}-\alpha_{2}^{\mathrm{s}}\right)+(1-2 y)\left(\alpha_{3}^{\mathrm{s}}-\alpha_{4}^{\mathrm{s}}\right)+\alpha_{\mathrm{c}}^{\mathrm{s}} \\
& \alpha_{1}^{\mathbf{s}}=\alpha_{\mathrm{AC}-\mathrm{BC}}^{\mathrm{s}}, \alpha_{2}^{\mathrm{s}}=\alpha_{\mathrm{AD}-\mathrm{BD}}^{\mathrm{s}}, \alpha_{3}^{\mathrm{s}}=\alpha_{\mathrm{AC}-\mathrm{AD}}^{\mathrm{s}}, \alpha_{4}^{\mathrm{s}}=\alpha_{\mathrm{BC}-\mathrm{BD}}^{\mathrm{s}}
\end{aligned}
$$

The spinodal equation defines the area of thermodynamical instability that is the inflexion line of this surface in relation to the $x$ and $y$ concentration axes (cf. Fig. 2). Obviously such a description of thermodynamical stability has a meaning either for homoepitaxial synthesis or homogenic nucleation in a solution.

During heteroepitaxy, the nature of atomical interactions is stimulated by the surface energy [5, 7]. Chemical potentials of anion-cation pairs are applied in the assumed models of thermodynamical balance, therefore the effect of interface on thermodynamical stability can be expressed through the electrical interactions.

It is acknowledged that during the initial stage of heteroepitaxy from a liquid phase, the interface lattice is coherent with the substrate lattice. Such a situation was schematically drawn in Fig. 1. The demand of coherency of interface and substrate lattices is equivalent to the demand of supplying the additional portion of energy for elastic deformation of solution elementary cell in accordance with the $G^{\text {ext }}$ curve (see Fig. 1). Assuming the ionic nature of III-V atomical bonds in the

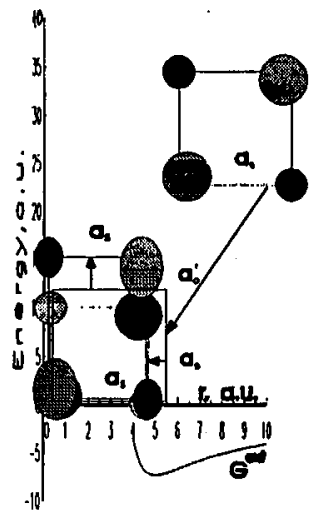

Fig. 1. Model of coherent deformation of interface lattice.

$\mathrm{A}_{x} \mathrm{~B}_{1-x} \mathrm{C}_{y} \mathrm{D}_{1-y}$ type solution, the change in the interface energy connected with the coherent shift of interface atoms can be written as

$$
\Delta G^{\text {ext }}=n N_{0}\left[\left(K q^{2} / 4 \pi \varepsilon_{0} a_{\mathrm{s}}-A / a_{\mathrm{s}}^{m}\right)-\left(K q^{2} / 4 \pi \varepsilon_{0} a_{0}-A / a_{0}^{m}\right)\right],
$$

where: $q$ - ion charge, $\varepsilon_{0}$ - electrical permittivity of vacuum, $K$ - Madelung's constant, $A, m$ - experimentally found coefficients, $a_{0}=a_{24}+b x+c y+d x y$; $b=a_{14}-a_{24}, c=a_{23}-a_{24}, d=a_{13}-a_{23}-a_{14}+a_{24}$. The typical value of $m$ coefficient for ionic crystals is $9 \div 11$. The relation between $A$ and $m$ can be found from the equality of forces in the stationary state of the matter. Then

$$
A=K q^{2} r_{0}^{m-1} / 4 \pi \varepsilon_{0} m,
$$


where $r_{0}$ - corresponds to the stationary position of the molecule (the equivalent of the lattice constant, $\left.a_{0}\right), a_{\mathrm{s}}-$ substrate lattice constant, $N_{0}$ - the Avogadro number.

Consequently, the excessive Gibbs' $\left(G^{m}\right)$ energy is rised by the increase in the interface energy. The spinodal equation, assuming the coherent coupling of phases, takes a form

$$
\left(\partial^{2} G^{*} / \partial x^{2}\right)\left(\partial^{2} G^{*} / \partial x^{2}\right)-\left(\partial^{2} G^{*} / \partial x \partial y\right)^{2}=0 ;
$$

where

$$
\begin{aligned}
& \left(\partial^{2} G^{*} / \partial x^{2}\right)=-\left(N_{0} K q^{2} / a_{\mathrm{s}}^{m} 4 \pi \varepsilon_{0} m\right)(m-1)(m-2)(b+d y)^{2} a_{0}^{m-3} \\
& -\left[2 N_{0} K q^{2} /\left(4 \pi \varepsilon_{0}\right)\right](b+d y)^{2} / a_{0}^{3} \\
& \quad+\left[2 N_{0} K q^{2} /\left(4 \pi \varepsilon_{0}\right)\right](b+d y)^{2} / m a_{0}^{3}+R T /[x(1-x)] \\
& \quad-2\left[y \alpha_{1}^{\mathrm{s}}+(1-y) \alpha_{2}^{\mathrm{s}}\right] \\
& \left(\partial^{2} G^{*} / \partial y^{2}\right)=-\left(N_{0} K q^{2} / a_{\mathrm{s}}^{m} 4 \pi \varepsilon_{0} m\right)(m-1)(m-2)(c+d x)^{2} a_{0}^{m-3} \\
& \quad-\left[2 N_{0} K q^{2} /\left(4 \pi \varepsilon_{0}\right)\right](c+d x)^{2} / a_{0}^{3}+\left[2 N_{0} K q^{2} /\left(4 \pi \varepsilon_{0}\right)\right](c+d x)^{2} / m a_{0}^{3} \\
& \quad+R T /[y(1-y)]-2\left[x \alpha_{3}^{s}+(1-x) \alpha_{4}^{s}\right], \\
& \left(\partial^{2} G^{*} / \partial x \partial y\right)=-\left(N_{0} K q^{2} / a_{s}^{m} 4 \pi \varepsilon_{0} m\right)(m-1) \\
& \quad \times\left[(m-2)(b+d y)(c+d x) a_{0}^{m-3}+d a_{0}^{m-2}\right] \\
& +N_{0} K q^{2} /\left(4 \pi \varepsilon_{0}\right)\left[d a_{0}+2(c+d x)(b+d y)\right] / a_{0}^{3} \\
& -N_{0} K q^{2} /\left(4 \pi \varepsilon_{0}\right)\left[d a_{0}-2(b+d y)(c+d x)\right] / m a_{0}^{3} \\
& +(1-2 x)\left(\alpha_{1}^{\mathrm{s}}-\alpha_{2}^{\mathrm{s}}\right)+(1-2 y)\left(\alpha_{3}^{\mathrm{s}}-\alpha_{4}^{\mathrm{s}}\right)+\alpha_{\mathrm{c}}^{\mathrm{s}} .
\end{aligned}
$$

\section{Results}

The results of calculations are depicted in Fig. 2. The calculations suggest the significant effect of parameters of interatomic interactions in solid solution on instability areas. It is particulary remarkable during reduction in solution temperature (see Fig. 2). Therefore for athermal [8] solutions, even under typical synthesis conditions of $\mathrm{Ga}_{x} \mathrm{In}_{1-x} \mathrm{P}_{y} \mathrm{As}_{1-y}$ alloys, difficulties in their synthesis should appear whereas the $\alpha_{i j}^{\mathbf{s}}$ parameters with the thermal dependence taken from [9] provide more reasonable results. In the present paper, the existence of spinodal decomposition without any contribution of the substrate was experimentaly confirmed for the $\mathrm{Ga}_{0.35} \operatorname{In}_{0.65} \mathrm{P}_{0.2} \mathrm{As}_{0.8}$ solution. The super-cooling of the homogenized solution at the temperature of $500 \mathrm{~K}$ caused the formation of nuclei of various compositions. 


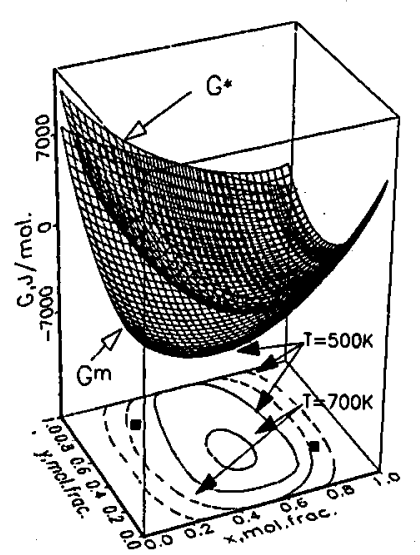

Fig. 2. Gibbs' surfaces of $\mathrm{Ga}_{x} \mathrm{In}_{1-x} \mathrm{P}_{y} \mathrm{As}_{1-y}$ solutions and spinodals with parameters: [8] - dashed line, [9] — solid line, a - experimental data of the present paper.

This fact was confirmed by the microbeam investigations of the super-cooled surface by means of the NL2001A(TESLA) spectrometer. The experimental data are depicted in Fig. 2. The presence of a larger number of phases on substrate surface of GaAs was not found after the contact with the same composition solution which correlates well with the above model of atomic interactions in the crystallization zone assuming coherent coupling of phases.

\section{References}

[1] V.V. Kuznetsov, P.P. Moskvin, V.S. Sorokin, in: Nierovnoviesnyje javlienia pri zidkostnoj heteroepitaksji poluprovodnikovych tviordych rostvorov, Metalurgia, Moskva 1991, (in Russian), p. 11.

[2] B. de Cremoux., J. de Physique 43, C-5-19 (1982).

[3] K. Onabe, Jpn. J. Appl. Phys. 21, 797 (1982).

[4] A.S. Jordan, M. Ilegems, J. Phys. Chem. Solids 36, Nr 4, 329 (1975).

[5] J.M. Olchowik, Pr. Nauk. P.L. 230, Fizyka 9, 57 (1993) (in Polish).

[6] B. de Cremoux, P. Hirtz, J. Ricciardi, in: Gallium Arsenide and Related Comp., 8th Institute Symposium Vienna, 1980, Inst. Phys. Conf. Ser., Vol. 56, Bristol 1981, p. 115.

[7] H. Sonomura, G. Sunatori, T. Miyauchi, J. Appl. Phys. 53, 5336 (1982).

[8] M.B. Panish, M. Ilegems., in: Progress in Solid State Chemistry, Ed. J.O. McCaldin, Pergamon Press, Oxford 1972, p. 39.

[9] A.T. Gorelonok, V.N. Mdivani, P.P. Moskvin, V.S. Sorokin, A.S. Usikov, J. Cryst Growth 60, 355 (1982). 